\title{
A NEW APPROACH TO SEGMENTATION BASED ON FUSING CIRCUMSCRIBED CONTOURS, REGION GROWING AND CLUSTERING
}

\author{
X. Muñoz, X. Cufí, J.Freixenet and J. Martí \\ Computer Vision and Robotics Group \\ Institute of Informatics and Applications. University of Girona. \\ Campus de Montilivi, 17071 Girona, Catalonia \\ \{xmunoz, xcuf, jordif, joanm\}@eia.udg.es
}

\begin{abstract}
One of the major problems in machine vision is the segmentation of images of natural scenes. This paper presents a new proposal for the image segmentation problem which has been based on the integration of edge and region information. The main contours of the scene are detected and used to guide the posterior region growing process. The algorithm places a number of seeds at both sides of a contour allowing starting a set of concurrent growing processes. A previous analysis of the seeds permits to adjust the homogeneity criterion to the region's characteristics. A new homogeneity criterion based on clustering analysis and convex hull construction is proposed.
\end{abstract}

\section{INTRODUCTION}

During the last few years many segmentation techniques, focusing on region-based and edge-based segmentation have been developed [1]. Unfortunately, these techniques fail to produce an accurate segmentation. Region-based techniques often fail to yield the desired structure due to the difficulty of choosing reasonable starting "seed" points and appropriate growing rules. Similarly, edge-based techniques often fail as a consequence of object similarity within the scene or indistinct boundaries among image objects.

Therefore, current trends in segmentation try to integrate several techniques in order to produce better results. Two possible ways of integration are possible: the first consists of refining the obtained segmentation using the results of another segmentation technique. The edge information, for example, can be used to refine a region segmentation process [2], or alternatively a region-based segmentation can be used to automatically add edge segments into a fully connected line-drawing [3]. The second way is based on integrating edge detection and region extraction in the same process [4].

Our approach follows the second approach using the edges of the image as a guide to orientate the later region growing process properly. Suppose a contour separates two regions. A set of germs is then located at both sides of the contour in order to know some features of the region. This prior knowledge allows the adjustment of the region growing algorithm and the resolution of a very common problem: the difficulty of establishing an adequate criterion of homogeneity. Classical algorithms base their decision of merging a region with a pixel on a homogeneity criterion which usually takes into consideration some statistical parameters on the regions. However, this criterion often may not be applicable to highly textured images or range images [5], because regions in such images usually have very different homogeneity behaviousr, as is depicted in fig. 1 .
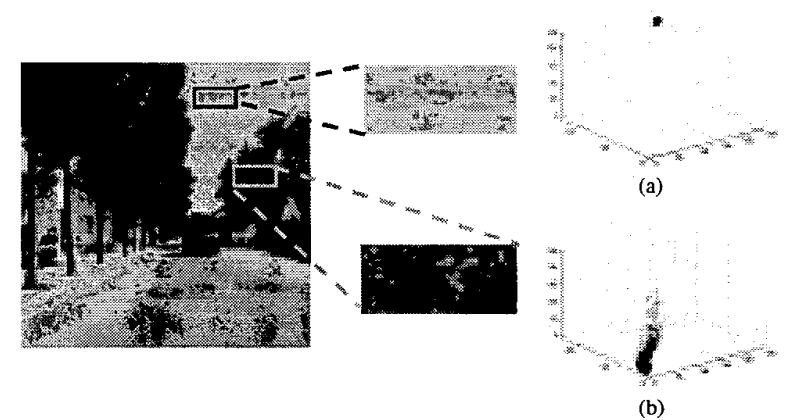

Figure 1. Two chromatic feature spaces corresponding to the imaged objects sky (a) and leaves (b), depicting different homogeneity behaviour.

Therefore, looking for a single criterion becomes an inadequate procedure. Our approach allows individual adjustment of the criterion to the region and the use of different criterion in each case. 


\section{OVERVIEW}

The scheme of the technique proposed consists of four basic steps (see fig.2). The first concentrates on main contour extraction and focuses on detecting the boundaries between the different regions of the image and filtering the edges with less relevance. The result is the extraction of the most relevant contours in the image." Getting these contours is a really difficult job but it is essential for further processing.

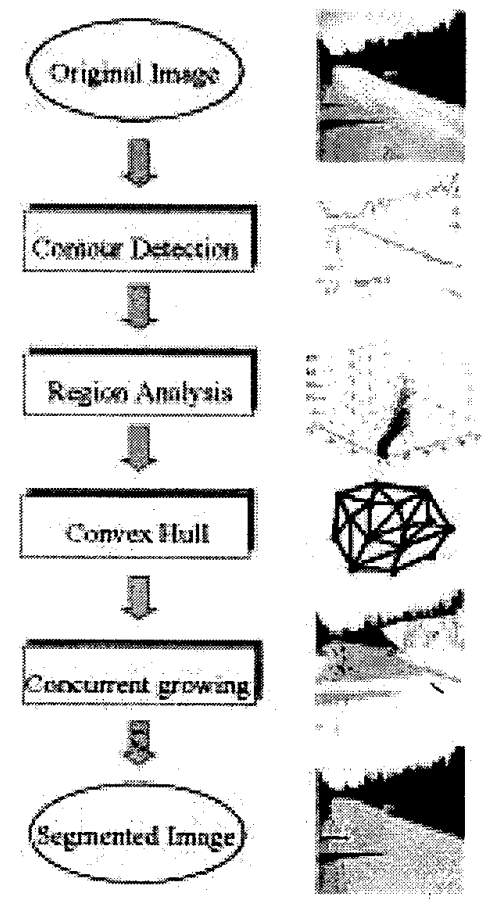

Figure 2. Scheme with the basic steps of the proposed technique.

At the beginning of the second step, a number of seeds are launched inside the region and used as samples. The seeds are then analysed using a hierarchical clustering algorithm with the objective of obtaining the clusters they generate on the chromatic space. This knowledge is further used in the third stage to construct the convex hull of each cluster. The convex hull establishes the shape of each cluster and is the base for the homogeneity criterion. The algorithm bases its decision to merge a pixel with the region on the relative placement of the pixel (insideoutside) in respect to the convex hulls associated with the samples of the region. Finally, in the fourth step, the germs simultaneously start a concurrent growth using the criterion established for each region. The result is a valid segmentation where each region corresponds to an object in the image, independently of the characteristics of the region.

\section{CONTOUR DETECTION}

The goal of the contour detection is to obtain a set of boundaries of the perceptively most significant regions, named the Circumscribed Contours of the image. The contour detection and extraction is realised according to the algorithm proposed in [6]. The method is based on the two most relevant properties presented by most significant frontiers of a determined scene: First, principal contours must have an outstanding length within the global frame of the image. Secondly, the regions separated by the contours should present some appreciable differences in chromatic and textural features.

\section{REGION ANALYSIS}

The contours obtained in the previous step represent the boundaries between regions. Every contour theoretically separates two adjacent regions in the image. The growing centres, called seeds, are chosen at each side and along the contour, as is illustrated in fig. 3a. From the edge results we suppose the seeds belonging to a determined side of the contour are associated to the same region. The seeds are used like samples of the region and are analysed in the chromatic space. Fig. $3 b$ shows a set of seed points, which represent the characteristics of the region located at one side of the contour, mapped into the RGB feature space.

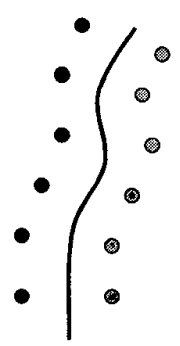

(a)

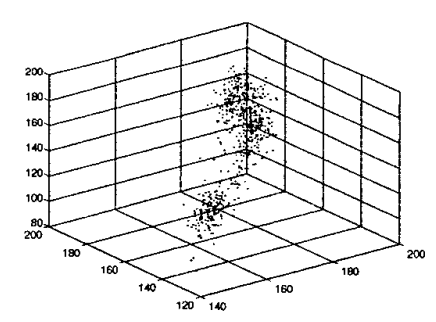

(b)
Figure 3. Two sets of seeds located at both sides of a contour (a), and the representation of one set in the chromatic space $\mathrm{RGB}$ in (b).

One essential characteristic of the distribution is the number of clusters which fit the samples. Each cluster has its own identity and must be considered individually. The region analysis tries to determinate the clustering in the region. A classic clustering algorithm is used with this objective. Hierarchical clustering constructs a hierarchical structure by iteratively merging clusters according to certain dissimilarity measure (concretely the Euclidean distance), starting from singleton elements until no further merging is possible. The identification of the number of clusters is realised using the sequence of clustering generated. An intuitive approach is to search for clusters 
which have a long lifetime. The lifetime of a cluster is defined as the absolute value of the difference between the proximity level which it is created and the proximity level which it is absorbed into a large cluster. The analysis of the seeds gives us the number of clusters and the seeds which are grouped in each cluster. This information is essential for the next step, where the most convenient criterion of homogeneity for the region is fixed.

\section{CONVEX HULL}

The criterion of homogeneity is established using the information from the analysis of the seeds. More concretely, this work remarks the necessity to consider the information relative to the shape of different clusters. This objective is achieved applying the technique of the convex hull.

The convex hull is a well-known concept in the computational geometry. By definition, the convex hull of a set of points $S$ is the smallest convex set containing $S$. The convex hull of a set of $\mathrm{N}$ points in three-dimensional space can be computed in optimal time $\theta(\mathrm{N} \operatorname{logN})$ [7]. The convex hull is used to obtain the shape of the clusters. Each cluster of points is treated individually and a convex hull is obtained for each one. This process defines the shape of each cluster in the chromatic space.

The decision of merging a pixel inside a region is based on the relative placement of the pixel in respect to the convex hulls associated with the region. If the pixel is inside any of the convex hulls, the pixel is merged into the region.

\section{CONCURRENT GROWING}

To ensure an efficient and easy implementation, a standard for distributed object oriented computing, CORBA [8], has been chosen. Following this specification, a set of objects-software can be programmed in heterogeneous programming languages, executed at several workstations with different operating systems which cooperate, in order to solve a concrete problem by using a common and simple mechanism.

Figure 4 shows a scheme in which the complete set of processes which participates in our proposal appear. Once the main process has detected the whole set of contours, it then creates two threads associated to each contour. These threads are dedicated to performing a specific region analysis at each side of the contour. Then, every thread launches a new set of threads which will perform the region growing process. These last processes, associated with the seeds, compete for the pixels of the image accessing in mutual exclusion. Finally, when all the processes have finished their growing, the algorithm begins to merge neighbouring regions.

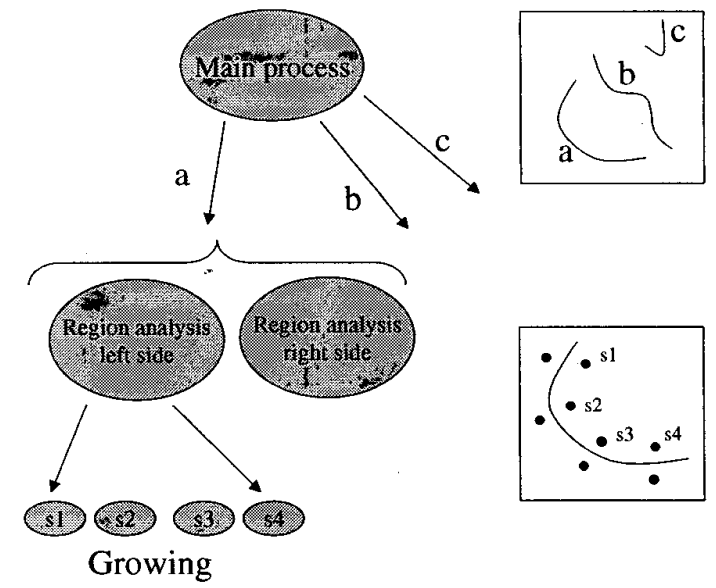

Figure 4. Scheme of the whole set of processes (threads) involved in the algorithm. Each $S_{i}$ is the task engaged to perform a region growing process starting at the pixel seed $i$.

\section{EXPERIMENTAL RESULTS}

The proposed segmentation algorithm has successfully segmented a wide range of natural images. Fig.4 illustrates the segmentation obtained in three representative images: a natural scene with a lake, a semi-structured zone of a road going through a forest and an urban location.

The results of the proposed segmentation technique (fig. 5b) are compared with the segmentation obtained by means of a classic region growing algorithm using the average as homogeneity criterion (fig. 5a). Note that the proposed technique achieves better results than traditional techniques on textured regions (forest the semi-structured image or leaves in the urban image), since the typical algorithm has over-segmented these regions. The use of a homogeneity criterion adjusted to the characteristics of a region permits grouping the pixels of the conceptual region inside a unique segmented region, and to solve the segmentation of highly textural images.

\section{CONCLUSIONS}

In this paper we have presented a method for the segmentation of natural images which integrates region and edge information. We have proposed a new homogeneity criterion for the typical region growing algorithm based on a previous analysis of a number of seeds located at the sides of the contours. This criterion is adjusted to the region's characteristics and is based on clustering analysis and construction of convex hulls of the seeds in chromatic space. Experimental results indicate that the proposed method provides much better results than traditional techniques in the segmentation of natural images. 

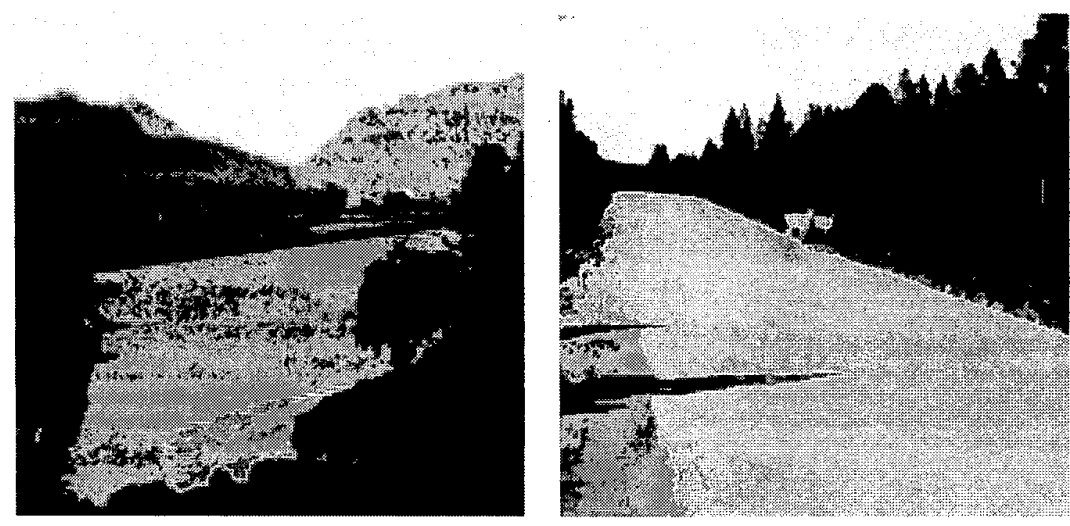

(a)
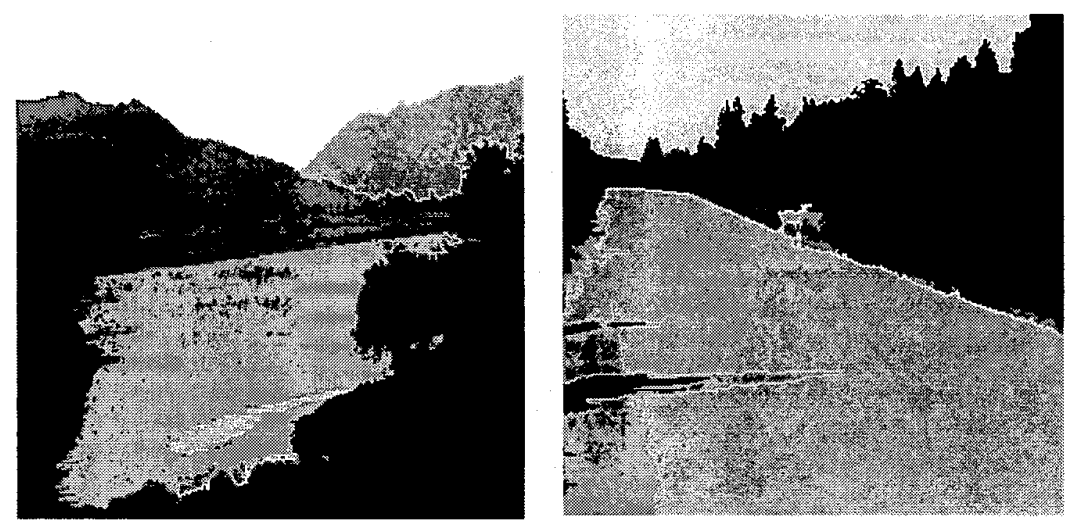

(b)
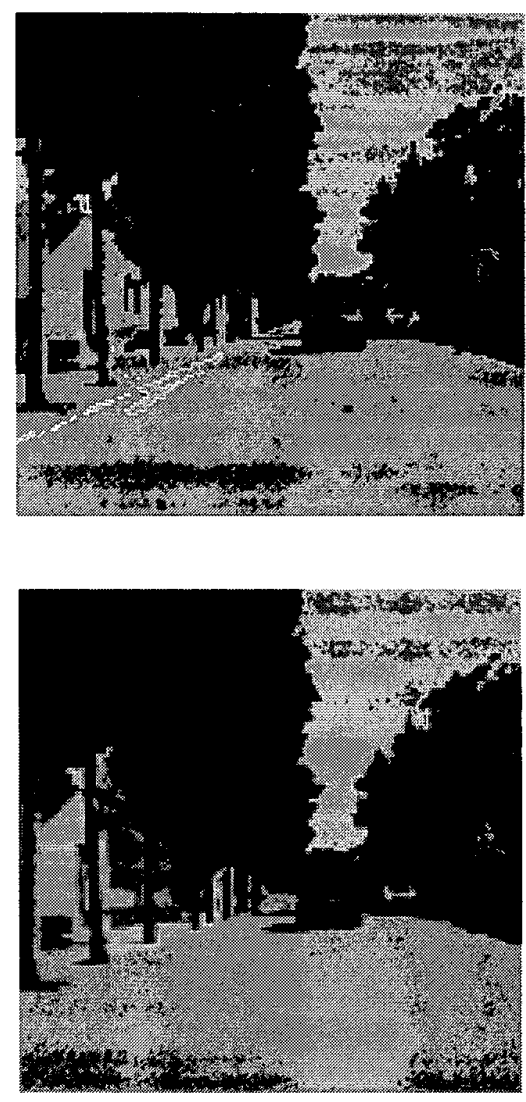

Figure 5. Results comparative. (a) Segmented image obtained by Region Growing (b) Segmented Image obtained by the proposed segmentation technique.

Moreover, the distributed implementation of this approach, which has been performed by using the standard CORBA, will ensure easy evolutions of the systems in the near future as well as the necessary current performance for parallel execution of tasks.

\section{REFERENCES}

[1] R. Haralick, L.Shaapiro, "Survey: Image Segmentation Techniques", Computer Vision Graphics, Image Processing, vol.29, pp.100-132, 1985.

[2] T. Pavlidis, Y.T. Liow, "Integration Region Growing and Edge Detection", IEEE Trans. on PAMI 12, No.3, pp 225-233, March 1990.

[3] E.J. Pauwels, P. Fiddelaers, L.J. Van Gool, "Autonomous Grouping of Contour-Segments Using an Adaptive RegionGrowing Algorithm“, Proceedings of ICPR'96, pp.586-590, 1996.
[4] J. P. Gambotto, "A new approach to combining region growing and edge detection", Pattern Recognition Letters 14, pp.869-875, 1993.

[5] R. Adams, L. Bischof, "Seeded Region Growing", IEEE Trans on PAMI 16, No.6, pp 641-647, June 1994.

[6] X. Cufí, A.Casals, "A Criterion for Circumscribed Contour Determination for Image Segmentation", $M V A^{\prime} 96$ IAPR Workshop on Machine Vision Applications, pp.309-312, Tokyo, November 1996.

[7] F.P.Preparata and S.J. Hong, "Convex Hulls of Finite Sets of Points in two and three Dimensions", Comm. ACM 2(20), pp.87-93, February 1977.

[8] S.Baker, CORBA Distributed Objects. Uning Orbix; In ACM Press. Addisson-Wesley; 1997. 